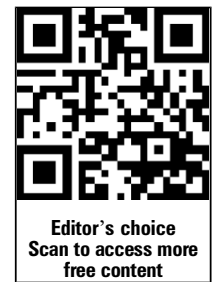

For numbered affiliations see end of article.

\section{Correspondence to} Dr Tjitske Kleefstra and Dr Annette Schenck, Department of Human Genetics, Radboud University Medical Centre,

PO Box 9101, Nijmegen 6500

$H B$, The Netherlands; t.kleefstra@gen.umcn.nl, a.schenck@gen.umcn.nl

MHW, BN, MF, AS and TK contributed equally.

Received 19 December 2012 Revised 28 March 2013 Accepted 8 April 2013 Published Online First 4 May 2013

\footnotetext{
To cite: Willemsen $\mathrm{MH}$, Nijhof $B$, Fenckova $M$, et al. $J$ Med Genet 2013;50: 507-514.
}

\title{
GATAD2B loss-of-function mutations cause a recognisable syndrome with intellectual disability and are associated with learning deficits and synaptic undergrowth in Drosophila
}

\author{
Marjolein H Willemsen, ${ }^{1}$ Bonnie Nijhof, ${ }^{1,2,3}$ Michaela Fenckova, ${ }^{1}{ }^{1,2,3}$ Willy M Nillesen, ${ }^{1}$ \\ Ernie M H F Bongers, ${ }^{1}$ Anna Castells-Nobau, ${ }^{1,2,3}$ Lenke Asztalos, ${ }^{4}$ Erika Viragh, ${ }^{5}$ \\ Bregje W M van Bon, ${ }^{1}$ Emre Tezel, ${ }^{1,2,3}$ Joris A Veltman, ${ }^{1,2,6}$ Han G Brunner, ${ }^{1,6}$ \\ Bert B A de Vries, ${ }^{1,6}$ Joep de Ligt, ${ }^{1,2,6}$ Helger G Yntema, ${ }^{1}$ Hans van Bokhoven, ${ }^{1}, 2,3$ \\ Bertrand Isidor, ${ }^{7}$ Cédric Le Caignec, ${ }^{7}$ Elsa Lorino, ${ }^{8}$ Zoltan Asztalos, ${ }^{4,5,9}$ \\ David A Koolen, ${ }^{1}$ Lisenka E L M Vissers, ${ }^{1,2,6}$ Annette Schenck, ${ }^{1,2,3}$ Tjitske Kleefstra ${ }^{1,3,6}$
}

\section{ABSTRACT}

Background GATA zinc finger domain containing $2 B$ (GATAD2B) encodes a subunit of the MeCP1-Mi-2/ nucleosome remodelling and deacetylase complex involved in chromatin modification and regulation of transcription. We recently identified two de novo loss-offunction mutations in GATAD2B by whole exome sequencing in two unrelated individuals with severe intellectual disability.

Methods To identify additional individuals with GATAD2B aberrations, we searched for microdeletions overlapping with GATAD2B in inhouse and international databases, and performed targeted Sanger sequencing of the GATAD2B locus in a selected cohort of 80 individuals based on an overlap with the clinical features in the two index cases. To address whether GATAD2B is required directly in neurones for cognition and neuronal development, we investigated the role of Drosophila GATAD2B orthologue simjang (simj) in learning and synaptic connectivity.

Results We identified a third individual with a $240 \mathrm{~kb}$ microdeletion encompassing GATAD2B and a fourth unrelated individual with GATAD2B loss-of-function mutation. Detailed clinical description showed that all four individuals with a GATAD2B aberration had a distinctive phenotype with childhood hypotonia, severe intellectual disability, limited speech, tubular shaped nose with broad nasal tip, short philtrum, sparse hair and strabismus. Neuronal knockdown of Drosophila GATAD2B orthologue, simj, resulted in impaired learning and altered synapse morphology.

Conclusions We hereby define a novel clinically recognisable intellectual disability syndrome caused by loss-of-function of GATAD2B. Our results in Drosophila suggest that GATAD2B is required directly in neurones for normal cognitive performance and synapse development.

\section{INTRODUCTION}

Intellectual disability (ID) is a group of disorders with an extremely heterogeneous clinical and genetic presentation. More than 500 ID genes have been identified and many more await discovery. This large number of ID genes is believed to converge onto a limited number of common underlying pathways and processes. ${ }^{1}$ Several ID genes encode proteins that are involved in chromatin modification. $^{12}$

Since the recent advent of next generation sequencing technology, whole exome sequencing (WES) has been successfully applied to the identification of genes for clinically established ID syndromes. ${ }^{3-6}$ In addition, family based WES was successful in elucidating causative de novo gene mutations in sporadic individuals who do not present with a recognisable syndrome..$^{7-9}$ However, exploration of the pathogenicity of mutations in genes not previously associated with ID remains challenging. To establish a conclusive molecular diagnosis, it is therefore required to detect mutations in the same candidate genes in additional individuals with similar phenotype. ${ }^{10}$ Moreover, additional evidence and insights into functional properties of novel genes are desirable and can be obtained through studies in cell or animal models.

By application of trio based WES, we recently reported the identification of 22 candidate genes for ID. ${ }^{8}$ Among these was the GATA zinc finger domain containing 2B (GATAD2B) gene (NM_020699.2). In this gene, we identified two loss-of-function mutations in two unrelated individuals (c.584dupT; p.(Asn195fs) and c.1408 C>T; p.(Gln470*)). GATAD2B encodes p66beta, a subunit of the transcription repressor complex MeCP1-Mi-2/nucleosome remodelling and deacetylase (NuRD), responsible for silencing of methylated DNA by nucleosome remodelling and histone deacetylation. ${ }^{11} 12$

Here, we report an additional individual with a disruptive GATAD2B mutation (c.565_566del; p. (Gln190fs)), representing the third loss-of-function mutation in this gene. The mutation was revealed by direct Sanger sequencing in a selected cohort of 80 individuals with overlapping features comprising ID, childhood hypotonia and an abnormal shape of the nose (including a tubular shape, prominent and 
broad base of the nose). In addition, we found a fourth individual with a $240 \mathrm{~kb}$ de novo microdeletion encompassing GATAD2B. Comparison of the phenotype of all four individuals revealed a remarkable overlap in clinical presentation.

Modelling of the Drosophila orthologue of GATAD2B loss-of-function confirms its role in cognition and shows a critical role of GATAD2B in synapse development. Together, our data establish a novel ID syndrome. It adds to the growing list of ID conditions that are caused by mutated genes involved in chromatin remodelling that can shed light onto the epigenetic control of cognition.

\section{METHODS}

\section{Patients}

Individuals 1 and 2 were ascertained through family based WES studies recently reported by our group ${ }^{7}$ (trio 4 in Vissers et al and trio 69 in Ligt et $a l^{8}$ respectively). The GATAD2B mutation in individual 1 was noticed in retrospect upon reanalysis of the sequencing data, after the detection of the mutation in individual 2 .

Subsequently, we searched for individuals with small microdeletions overlapping with GATAD2B in our inhouse database and international databases, including the database of the European Cytogeneticists Association Register of Unbalanced Chromosome Aberrations (ECARUCA) and the Database of Chromosomal Imbalance and Phenotype in Humans using Ensembl Resources (Decipher).

In addition, we selected a cohort of 80 individuals guided by phenotypic overlap with individuals 1 and 2 . This cohort was selected based on the presence of severe ID (IQ $\leq 50)$, limited speech ability, childhood hypotonia and facial features including abnormal shaped nose (large/prominent nose, full nose tip, tubular shaped/pear-shaped nose, broad base of the nose). All individuals had been referred to the Department of Human Genetics of the Radboud University Medical Centre in Nijmegen, the Netherlands, for genetic diagnostic evaluation of unexplained ID/developmental delay. Their parents/legal representatives consented to this study. The study was approved by the local ethical committee.

\section{Mutation screening}

Targeted Sanger sequencing of GATAD2B (NM_020699.2) was performed using standardised methods. Primers are available upon request. The mutation in individual 4 and the low grade mosaic in her mother were confirmed with a second independent primer pair.

\section{Fly stocks and maintenance}

Fly stocks were kept on standard Drosophila diet (cornmeal/ sugar/yeast) at $25^{\circ} \mathrm{C}$ and $45 \%-60 \%$ humidity at $12 \mathrm{~h}$ light-dark cycle. Flies were reared at $25^{\circ} \mathrm{C}, 70 \%$ humidity for habituation experiments and real-time PCR, and at $28^{\circ} \mathrm{C}, 60 \%$ humidity to evaluate synapse morphology. Simjang (Simj) (CG32067) is the Drosophila orthologue of GATAD2B (Ensemble, see also Results section). An inducible RNA interference (RNAi) line against simj (vdrcKK100285, a line with the highest specificity score, $s 19=1)$ and its corresponding control line (vdrcKK60100) were obtained from Vienna Drosophila RNAi Center (http://www. vdrc.at). RNAi was induced by the UAS-Gal4 system using a panneuronal elav-Gal4 driver or a ubiquitous actin-Gal4 driver obtained from the Drosophila Bloomington Stock Center (http://flystocks.bio.indiana.edu/). Line $w^{1118} ; 2 x G M R-w I R$; elav-Gal4, UAS-Dicer2 used in habituation experiments was produced by recombination of two independent insertions of GMR-wIR on chromosome II and recombination of one copy of
elav-Gal4 and UAS-Dicer2 insertions on chromosome III. The $\mathrm{w}^{1118}$; UAS-Dicer2; elav-Gal4 driver was used to study synapse morphology, and a w ${ }^{1118}$; actin-Gal4/CyOGFP driver to generate homogeneous knockdown material for real-time PCR.

\section{Light-off jump reflex habituation}

The light-off jump reflex habituation assay was performed as previously described ${ }^{13}$ with minor adaptations to the protocol. Briefly, 3-7-day-old individual male flies were tested for jump response in two independent 16-unit light-off jump reflex habituation systems. A total of 32 flies (16-flies/system) were simultaneously exposed to series of 100 short $(15 \mathrm{~ms})$ light-off pulses with a $1 \mathrm{~s}$ interval between the pulses. The noise amplitude of wing vibration following every jump response was recorded for $500 \mathrm{~ms}$ after the start of pulse and a carefully chosen threshold was applied to distinguish the jump response. Data were collected and analysed by custom-made Labview Software (National Instruments). High initial jumping response to light-off pulse decreased with growing number of trials and flies were considered habituated when they failed to jump in five consecutive trials (non-jump criterion). Habituation was scored as the number of trials required to reach the non-jump criterion (Trials To Criterion (TTC)). Mean TTC values of eight independent groups of 16 flies (tested on four different days) were compared with mean TTC values of control flies using one-way Analysis of variance (ANOVA) with correction for different experimental day and system.

\section{Drosophila synapse morphology}

Type 1 b neuromuscular junctions (NMJs) at muscle 4 were analysed after dissection of L3 larvae and fixation in 3.7\% paraformaldehyde (PFA) for $30 \mathrm{~min}$. Preparations were colabelled for bruchpilot (brp) and discs large 1 (dlg1). Brp was visualised using the primary antibody nc82 (1:125) (Developmental Studies Hybridoma Bank, University of Iowa) applied overnight at $4^{\circ} \mathrm{C}$, and a secondary Alexa 488-labelled goat-antimouse antibody (1:500) (Invitrogen). Discs large was visualised using the primary antibody anti-dlg1 (1:25) (Developmental Studies Hybridoma Bank) in combination with the Zenon Alexa Fluor 568 Mouse IgG1 labelling kit (Invitrogen). NMJ pictures were obtained using a Leica automated brightfield multi-colour epifluorescent microscope. Individual synapses were imaged and the muscle area, NMJ area, perimeter, length, branching pattern and amount of active zones were quantitatively assessed using an inhouse developed macro.

\section{Analysis of simj mRNA levels by real-time PCR}

Total RNA from 3rd instar larvae was isolated using RNeasy Lipid Tissue Mini Kit (Qiagen). RNA was treated with DNase (DNAfree Kit, Ambion). First strand cDNA synthesis was performed using the Moloney Murine Leukemia Virus (M-MLV) Reverse Transcriptase (Life Technologies) and oligo(dT) primer. Gene expression was analysed by real-time PCR (7900HT Fast Real-Time PCR system, Applied Biosystems). PCR reactions were performed in a volume of $25 \mu \mathrm{l}$ containing $150 \mathrm{nM}$ primers and GoTag Green Mastermix (Promega). Primer sequences used for amplification of simj were 5'-CAGCACCATTCCGTTGTG-3' (forward primer) and 5'-GCCTTGAGTGCCTTCTTCAC-3' (reverse primer). A PolII amplicon was used as an internal standard. PollI primer sequences were $5^{\prime}$-TCAGAGTCCGCGTAA CACC-3' (forward primer) and 5'-TGGTCACAAGTGGCTTCA TC-3' (reverse primer). 


\section{RESULTS}

\section{Clinical phenotype description}

We here report the detailed clinical description of four affected individuals. Individual 1 (figure 1A-D) was born after 36 weeks and 5 days of pregnancy with a normal birth weight and no major complications upon delivery. At 2 years of age, her parents consulted a paediatrician because of global developmental delay. She could sit and crawl at the age of 16 months and stand at the age of 18 months. Hypotonia was noticed. After the age of 2 years she spoke her first words. An ophthalmologist was consulted because of strabismus. At the age of 3 years and 8 months she was able to speak about 30 single words, but her comprehension of language was at a higher level. She had a normal height $(100 \mathrm{~cm}, 30$ th centile) and head circumference $(47.5 \mathrm{~cm}, 10$ th centile). Her facial features included thin blond hair, narrow palpebral fissures, periorbital fullness, a tubular shape of the nose with full nasal tip, a short philtrum, thin upper lip, broad mouth and grimacing facial expression. Three years later, at the age of 6 years and 7 months she had severe developmental delay with verbal skills more severely impaired than motor skills. Her height was normal $(118.5 \mathrm{~cm}, 16$ th centile) and she had a low to normal head circumference $(48 \mathrm{~cm}, 2$ nd centile). Long fingers and toes with broad distal phalanges were observed.

Individual 2 (figure 1E-H) was born with intrauterine growth retardation. Her birth weight was $2780 \mathrm{~g}$ at 42 weeks of pregnancy $(<2$ nd centile). At 3 months of age a developmental delay was noticed. She could walk independently at the age of 3 years. From 8 years on, she could speak single words. Behaviour was characterised by tics and wandering during the night. Her pain threshold was elevated. Vision was impaired due to hypoplasia of the optic nerve. Upon clinical evaluation at the age of 34 years she was diagnosed with severe ID. At that time, height and head circumference were normal (both $>16$ th centile). She had thin blond hair, deeply set eyes, narrow and upward slanting palpebral fissures, strabismus, a tubular shape of the nose with broad nasal tip, and a large mouth with short philtrum and thin upper lip. She grimaced. Her fingers were long, thin and slightly tapering.

Individual 3 (figure $1 \mathrm{I}, \mathrm{J}$ ) was born after an uncomplicated pregnancy of 40 weeks duration, with a low-normal birth weight

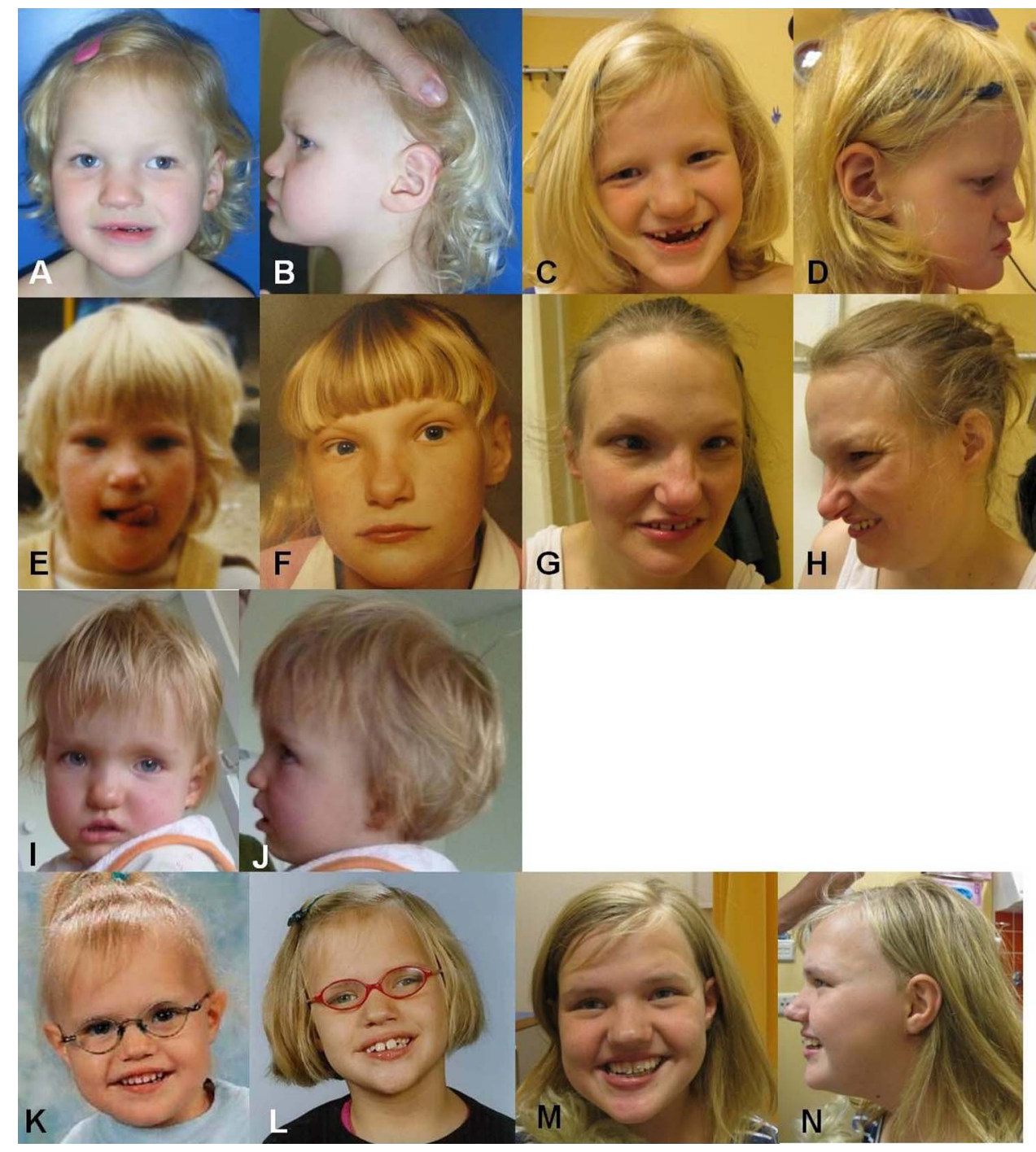

Figure 1 Facial features of individuals 1-4. Individual 1 at the age of 3 years and 8 months (A and B) and 6 years and 7 months (C and D). Individual 2 at the age of 5 years $(E)$, at older childhood age $(F)$ and at age 35 years $(\mathrm{G}$ and $H)$. Individual 3 at the age of 3 years (I and J), and individual 4 at the age of 12 years $(\mathrm{M}$ and $\mathrm{N})$ and younger ages $(\mathrm{K}$ and $\mathrm{L})$. Note the similarity in facial features, comprising thin, blond hair, broad forehead, hypertelorism, periorbital fullness, deeply set eyes and narrow palpebral fissures, a short philtrum, large, tubular shaped nose with broad nasal tip, broad mouth, strabismus, and remarkable grimacing facial expression in individuals 1, 2 and 4. Access the article online to view this figure in colour. 
and a normal head circumference. In the neonatal period she presented with hypotonia and feeding difficulties. Her psychomotor development was delayed. She learnt to walk without support at the age of 2 years and 9 months. At the age of 3 years speech was severely delayed with only two to three disyllabic words. Her behaviour was characterised by low frustration tolerance. Medical problems included hypermetropia and strabismus and she had shown one episode of absence epilepsy. At the age of 3 years she had a height of $90 \mathrm{~cm}$ (5th centile), and a head circumference of $51 \mathrm{~cm}$ (84th centile). Facial dysmorphism included hypertelorism, a broad forehead, a broad and flat nasal bridge and a full square tip of the nose. She had thin, blond hair.

Individual 4 (figure $1 \mathrm{~K}-\mathrm{N}$ ) was born after 42 weeks of pregnancy with a normal birth weight. Birth was uncomplicated. She was hypotonic and passive in the neonatal period. From the beginning on, psychomotor development was delayed with poor contact making. She learned to walk from the age of 2 years and started to speak during her third year. Her speech slowly developed, and at the age of 12 years she could only speak six single words. Her behaviour was characterised by hyperactivity, inappropriate laughter, obsession for shiny and reflecting objects and mild self-mutilation. Melatonin treatment was given for sleep problems. Medical problems included intermittent divergent strabismus and hypermetropic astigmatism, and persistent constipation. Around the age of 2-3 years absence epilepsy was suspected, but electroencephalography revealed no abnormalities. Upon physical examination at the age of 12 years and 2 months she had a height of $156 \mathrm{~cm}$ (50th centile), a weight of $43 \mathrm{~kg}$ (16th-50th centile) and a head circumference of $56.2 \mathrm{~cm}$ (80th-90th centile). Facial dysmorphism included a broad forehead, a broad nasal bridge with full nose tip, a broad mouth with wide-spaced central incisors, short philtrum and long palpebral fissures. She had thin, blond hair. Her fingers were long and she had fleshy hands.

\section{Mutation detection}

Individual 1 showed no abnormalities on $250 \mathrm{~K}$ Singly Nucleotide Polymorphism (SNP) array analysis, screening for Fragile X syndrome, a metabolic screen in blood and urine, and MRI imaging of the brain. Family based WES revealed a de novo frameshift mutation in GATAD2B (c.584dup; p. (Asn195fs); figure 2A). This mutation is located in the central part of the gene close to the conserved region 1 (CR1) domain (figure 2D). ${ }^{8}$ Likewise, individual 2 did not show any abnormality on $250 \mathrm{~K}$ SNP array analysis and a metabolic screen. Methylation tests of the Angelman syndrome related region were normal. Family based exome sequencing revealed a de novo mutation in GATAD2B (c.1408 C>T; p.(Gln470*); figure 2B). This mutation is located in the conserved region 2 (CR2) domain (figure 2D). ${ }^{8}$

B

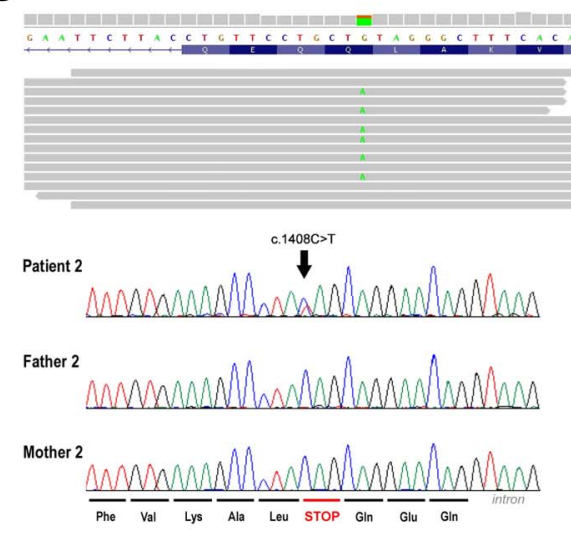

Figure 2 BAM files and results of Sanger sequencing in individuals 1, 2 and 4 and their parents. Validation and de novo testing for the candidate de novo mutations in GATA zinc finger domain containing 2B (GATAD2B) in individuals 1 and 2, and direct sequencing of the 11 coding exons of GATAD2B in the cohort of individuals with phenotypic overlap, including individual 4, were performed using standard Sanger sequencing approaches. Primer sequences and PCR conditions are available upon request. (A and B) show the BAM files and the Sanger sequences of individuals 1 and 2, and (C) Sanger sequences of individual 4. Sequences of both parents of individuals 1 and 2 and the mother of individual 4 are shown as well. The mother of individual 4 carries the mutation in low mosaic state $(<10 \%)$. (D) illustrates the relative positions of the mutations of individuals 1,2 and 4 in the GATAD2B protein. CR1, conserved region 1; CR2, conserved region 2. Access the article online to view this figure in colour. 
Individual 4 was previously investigated for mutations in the genes FMR1, MECP2, TCF4, RAI1 and UBE3A, all showing normal results. Methylation tests of the Angelman syndrome related region and a metabolic screen gave also normal results. Conventional G-banded karyotyping yielded a maternally-inherited apparently balanced translocation between chromosomes 1q21.3 and 9q13. Further 250K SNP array analysis did not show any imbalances of these regions. Targeted sequencing of GATAD2B based on phenotypic overlap with the first two individuals led to the identification of a frameshift mutation (c.565 566del; p.(Gln190fs); figure 2C). This mutation is located at the border of the CR1 domain (figure 2D). The healthy mother carried the GATAD2B mutation in a low mosaic level, which was estimated to be below $10 \%$ in peripheral blood lymphocytes (figure 2C). The mother is highly educated and works as a social worker. She has no facial dysmorphism.

\section{Copy number variation detection}

Array comparative genomic hybridisation (CGH) analysis in individual 3 by a $60 \mathrm{~K}$ Agilent array, using the International Standards for Cytogenomic Arrays (ISCA) design, revealed a $240 \mathrm{~kb}$ de novo deletion of chromosome 1q21.3 encompassing 10 genes, including GATAD2B (chr1:153 893 110$154132780, \mathrm{Hg} 19)$. The distal breakpoint disrupts GATAD2B. The deleted region encompasses no known disease associated genes. The deletion was confirmed by Fluorescent in situ Hybridisation (FISH) with the RP11-422P24 probe. Segregation analysis in the parents was done by FISH analysis and showed that the deletion had occurred de novo.

\section{Analysis of GATAD2B orthologue in Drosophila}

To obtain independent evidence for the involvement of GATAD2B in the ID phenotype of the described individuals, we decided to study its function in an intact nervous system, using Drosophila as a model. The Drosophila genome contains a single orthologue of the two closely related human GATAD2A and GATAD2B genes, named simjang (simj). Drosophila simj has been shown to modify Wnt signalling and, like both its human counterparts, to associate with the MeCP1-Mi-2-NuRD

\section{Neuronal knockdown of Drosophila GATAD2B orthologue results in impaired learning}

Null mutations of Drosophila sim $j$ have previously been reported to be lethal, ${ }^{14}$ which precluded assessment of cognitive function in adult flies. We therefore targeted expression of simj specifically repressor complex. ${ }^{14}$

in Drosophila neurones using the UAS-Gal4 system in combination with inducible $\mathrm{RNAi}^{15}$ and examined the role of $s i m j$ in habituation. Habituation is a form of non-associative learning where the probability of a behavioural response decreases with repeated presentations of a stimulus. ${ }^{16}$ Habituation was previously shown to be defective in classic learning and memory mutants and in a Drosophila model of ID. ${ }^{2} 1718$

To induce a panneuronal knockdown of simj, flies carrying an inducible UAS-RNAi insertion against simj (vdrcKK100285) were mated to flies carrying the neuronal promoter element elav-Gal4 driver. The promoter line further carried one copy of UAS-dicer2 to enhance the RNAi-mediated knockdown and two insertions of GMR-wIR to reduce the eye colour as required in our assay. Male progeny of knockdown (simj-RNAi) and control flies was exposed to 100 short light-off stimuli (trials) at $1 \mathrm{~s}$ inter-trial intervals and scored for a jump response. Flies were considered to have habituated once they failed to jump in five consecutive trials (no-jump criterion). Habituation was scored as the number of trials required to reach the no-jump criterion (TTC). Both genotypes showed wt-like initial jump responses. Control flies quickly habituated to the light-off stimuli and reduced jumping. We found that simj-RNAi flies habituate slower and maintain a higher jump response throughout the entire course of the experiment (figure 3A). The mean TTC of simj-RNAi flies was 1.7-fold higher compared with their genetic background controls (figure $3 \mathrm{~B}, \mathrm{p}=0.007$ ), validating a significant deficit in habituation.

\section{GATAD2B is required for synaptic development in Drosophila}

Synaptic connectivity is essential for learning and for other cognitive processes. ${ }^{1}$ We therefore addressed a possible function of simj in synaptic development of the larval NMJ, a wellestablished synaptic model system that shares major features with central excitatory synapses in the mammalian brain ${ }^{19}$ and has been successfully used to investigate human ID disorders. ${ }^{20-22}$ As in our habituation experiment, we investigated the Drosophila NMJ architecture upon panneuronal knockdown of simj. The synaptic and subsynaptic organisation was visualised by coimmunolabelling against $\operatorname{dlg} 1(\alpha-\mathrm{dlg} 1)$, a major scaffolding component of larval NMJs and member of the membrane-associated guanylate kinase subfamily, and anti-brp ( $\alpha$-brp, nc82), an integral part of active zones. $^{22}$

Neurone-specific simj knockdown resulted in an NMJ undergrowth phenotype (figure 4A,B), with a consistent decrease in synaptic area $(\mathrm{p}=0.0028)$, perimeter $\left(\mathrm{p}=9.5 \mathrm{e}^{-005}\right)$ and length

A

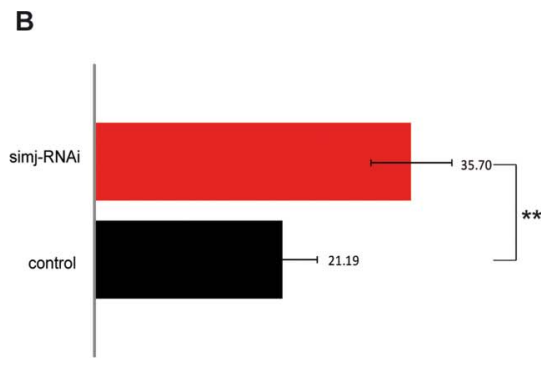

B

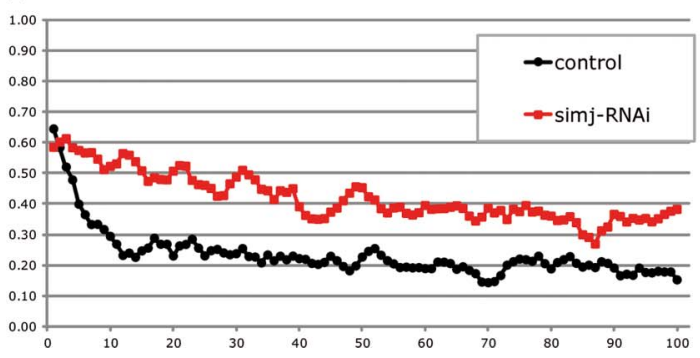

Figure 3 Impaired habituation of neurone-specific knockdown of Drosophila GATA zinc finger domain containing 2B orthologue Simjang (simj).

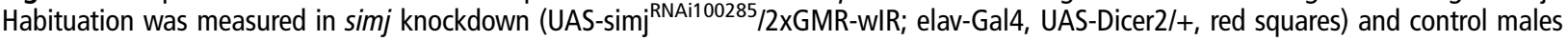
(2xGMR-wIR/+; elav-Gal4, UAS-Dicer2/+, black circles). Jump response was induced by repeated light-off pulses for 100 trials with $1 \mathrm{~s}$ inter-trial interval. y Axis represents the average jump response whereas $x$ axis represents number of trials $(A)$. Habituation was significantly slower for simj knockdown flies (red bar, Trials To Criterion $(\mathrm{TTC})=35.70$ ) than for control flies (black bars, TTC=21.17). ${ }^{* *}$ ) indicates a significant difference $(p<0.01)$ based on one-way ANOVA (B). RNAi, RNA interference. Access the article online to view this figure in colour. 

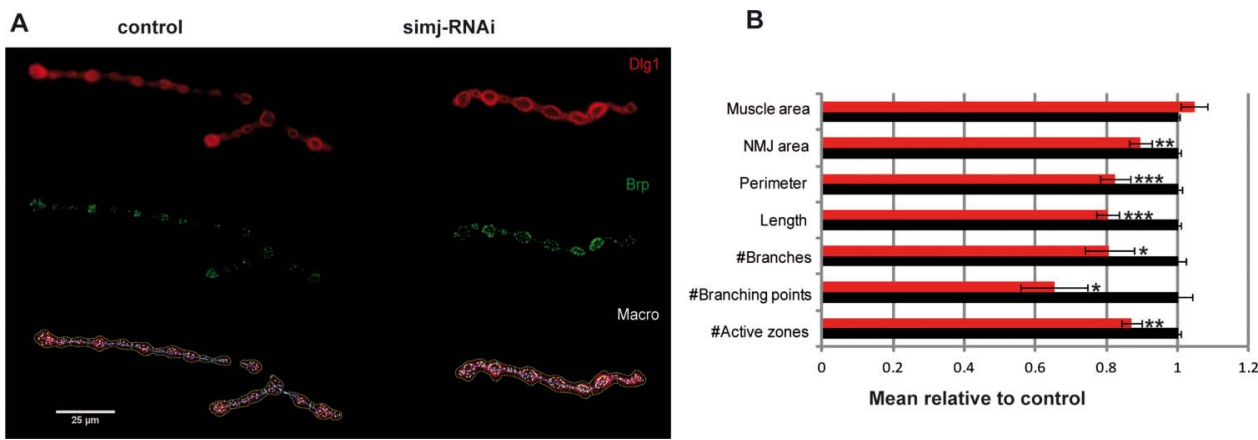

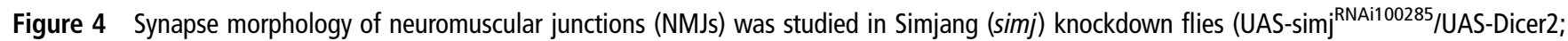
elav-Gal4/+) (A). NMJs were visualised with coimmunolabelling against discs large $1(A$, upper panel) and brp (A, middle panel) and quantitatively assessed by computer-assisted analysis ( $A$, lower panel and B). Simj-RNA interference (RNAi) NMJs show a decrease in NMJ area ( $n=27, p=0.0028)$, perimeter $\left(n=27, p=9.5 e^{-005}\right)$, length $\left(n=28, p=5.7 e^{-008}\right)$, the amount of branches $(n=28, p=0.041)$ and branching points $(n=28, p=0.021)$ and the amount of active zones $(n=25, p=0.0010)$. The area of the muscle remained normal $(n=28, p=0.077)$. For this plot, red bars represent the mean of each parameter of Simj-RNAi NMJs normalised by the mean of each parameter of the control set (black bars). Error bars indicate the normalised standard error of the mean, $p, p$ values (two-sided $t$ test); $n$, number of quantified NMJs. $\left({ }^{*}\right)$ indicates a significant difference $(p<0.05),\left({ }^{* *}\right)$ indicates a significant difference $(p<0.01),\left({ }^{* *}\right)$ indicates a significant difference $(p<0.001)$. Access the article online to view this figure in colour.

$\left(\mathrm{p}=5.7 \mathrm{e}^{-008}\right)$. Sim $j$-RNAi synapses also exhibited a lower number of branches $(\mathrm{p}=0.041)$ and branching points $(\mathrm{p}=0.021)$. Last, sim $j$-RNAi synapses showed a lower number of active zones $(p=0.001)$, revealing a reduced number of presynaptic sites for neurotransmitter release. Muscle size was not affected, which excludes a general growth/developmental problem. We validated the potency of the used RNAi line to induce simj knockdown by real-time PCR. The level of simj mRNA was efficiently downregulated to $29 \%$ upon induction by a ubiquitous actin-Gal4 driver ( $p=0.0024$; two-tailed t test). We conclude that reduced levels of the GATAD2B orthologue simj affect learning and synapse development.

\section{DISCUSSION}

In this study, we report a novel clinically recognisable syndrome characterised by severe ID, limited speech, childhood hypotonia, thin hair and recognisable facial features, including a typical, tubular shaped nose with broad tip, deeply set eyes, broad forehead, short philtrum, broad mouth, grimacing facial expression, strabismus and long fingers caused by haploinsufficiency of GATAD2B. We recently identified by family based exome sequencing disruptive GATAD2B mutations in two individuals with overlapping phenotype. ${ }^{8}$ We traced a third individual with a small microdeletion encompassing GATAD2B showing a very similar phenotype. Though we cannot fully exclude a contribution of the nine other genes in the deleted region of this individual, the important phenotypic overlap with individuals 1 and 2 highly suggests that deletion of GATAD2B is the major causal factor in the phenotype of this individual. Moreover, this further confirms the role of GATAD2B in a recognisable syndrome with ID represented by the present individuals. To ultimately further establish a novel ID syndrome, we selected a cohort of 80 unsolved similarly affected individuals based on clinical overlap. Targeted Sanger sequencing of the GATAD2B locus revealed an additional patient carrying a frameshift GATAD2B mutation, presumably causing loss-of-function.

We noticed that $G A T A D 2 B$ is located in the chromosomal region $1 \mathrm{q} 21.3$ where the breakpoint of the balanced translocation that was previously found in individual 4 and her healthy mother is also located. Although this finding might be coincidental, it raised the question whether the mother carried a mosaic translocation and whether the translocation breakpoint predisposed to the deletion of two base pairs in GATAD2B. We therefore performed additional FISH analysis with the region 1q23.1 specific probe RP11-216N14 that covers the GATAD2B gene. This showed in all 29 evaluated cells a normal pattern, indicating that the translocation breakpoint is located distally from the GATAD2B region. Therefore, we concluded that this does not support a correlation between the mutation in GATAD2B and the balanced translocation.

Complementary functional studies of the Drosophila GATAD2B orthologue sim $j$ demonstrated a role for the evolutionarily conserved GATAD2B gene family in neurodevelopmental processes. The identified habituation defect in simj-RNAi flies demonstrates that the GATAD2B orthologue is required for non-associative learning. Furthermore, evaluation of synaptic morphology revealed an NMJ undergrowth phenotype with reduced number of active zones, suggesting a critical role for simj and GATAD2B in synaptic growth and function. Whether these may contribute to or cause the observed learning defects in flies and cognitive deficit in the humans remains to be determined.

Mutations in several recently identified genes involved in chromatin modification give rise to ID syndromes, such as the 17q21.31 microdeletion/Koolen-De Vries syndrome (KDVS (MIM 610443)), ${ }^{23}{ }^{24}$ Coffin-Siris syndrome $^{13} \quad 25$ (CSS (MIM135900)), Nicolaides-Baraitser syndrome 27 (NCBRS (MIM 601358)), Kleefstra syndrome ${ }^{28}$ (MIM 610253), Wiedemann-Steiner syndrome ${ }^{29}$ (WDSTS (MIM 605130)) and Ohdo syndrome Say-Barber-Biesecker variant ${ }^{30}$ (SBBYSS (MIM 603736).

GATAD2B encodes p66beta, which is a subunit of the transcription repressor complex MeCP1-Mi2-NuRD that silences methylated DNA by nucleosome remodelling and histone deacetylation. This enzyme complex also comprises its close paralogue p66alpha (encoded by GATAD2A), the histone deacetylases HDAC1 and HDAC2, two histone binding proteins RbAp46 and RbAp48, the methyl binding domain protein 3 (MBD3), two histone modifier proteins, MTA1 and MTA2, and nucleosome remodelling factor Mi-2. ${ }^{11}$ It is thus conceivable that additional genes from the MeCP1-Mi2-NuRD complex, such as GATAD2A, are involved in phenotypes overlapping with the here defined novel syndrome caused by GATAD2B mutations. P66beta and its paralogue, p66alpha, function synergistically and recruit the Mi-2-NuRD complex to its target sites. They interact with methyl-CpG bound MBD2 and with 
non-acetylated histones to assemble in the so-called MeCP1 repressor complex. ${ }^{11}$ It has been shown that p66alpha and p66beta, and particularly their highly conserved CR1 and CR2 domains, are crucial for complex formation and mediated gene silencing. ${ }^{12}$ Furthermore, homozygous loss-of-function of p66alpha in mice resulted in an embryonic lethal phenotype with severe global malformations, growth retardation and necrosis. Heterozygous mice were viable and appeared normal, but detailed (neurological) phenotyping was not performed. ${ }^{31}$

We recently used the powerful strategy of combining human genetic studies and Drosophila modelling to provide a novel chromatin remodelling module that underlies Kleefstra syndrome spectrum. ${ }^{2}$ Similar to that, we find a strikingly high conservation between human and Drosophila NuRD complexes, with all complex components being present in fly. ${ }^{32}$ As additional variants in human NuRD complex genes will arise, it will be straightforward to validate their significance. Our data add $G A T A D 2 B$, and possibly the whole MeCP1-Mi2-NuRD complex, to the growing list of ID genes involved in chromatin remodelling.

\author{
Author affiliations \\ 1 Department of Human Genetics, Radboud University Medical Centre, Nijmegen, \\ The Netherlands \\ ${ }^{2}$ Nijmegen Center for Molecular Life Sciences, Radboud University Medical Centre, \\ Nijmegen, The Netherlands \\ ${ }^{3}$ Donders Institute for Brain, Cognition and Behavior, Radboud University Medical \\ Centre, Nijmegen, The Netherlands \\ ${ }^{4}$ Department of Genetics, Aktogen, University of Cambridge, Cambrigde, UK \\ ${ }^{5}$ Institute of Biochemistry, Biological Research Center, Hungarian Academy of \\ Sciences, Szeged, Hungary \\ ${ }^{6}$ Institute for Genetic and Metabolic Disease, Radboud University Medical Centre, \\ Nijmegen, The Netherlands \\ ${ }^{7}$ Service de Génétique Médicale, CHU Nantes, Nantes, France \\ ${ }^{8}$ Service de Pédiatrie, CHU Nantes, Nantes, France \\ ${ }^{9}$ Aktogen Hungary Ltd., Biological Research Center, Hungarian Academy of Sciences, \\ Szeged, Hungary
}

Acknowledgements We thank the participating individuals and their families. We also thank Suzanne Vloet-Keijzer and Martine Ruiterkamp for their technical assistance with the targeted Sanger sequencing.

Contributors The study was designed and the results were interpreted by MHW, TK, BN, MF, ZA, LA, AC-N, EV, AS, LELMV, HGB, HvB and JAV. Subject ascertainment and recruitment were carried out by MHW, TK, BWMvB, DAK, EMHFB, BI, CLC, EL and BBAdV. Sequencing and genotyping were carried out and interpreted by WMN, HGY, LELMV, JdL and JAV. The manuscript was drafted by MHW, BN, MF, TK and AS. All authors contributed to the final version of the paper.

Funding This work was supported by grants from the Consortium 'Stronger on your own feet' to TK and MHW, The Netherlands Organization for Health Research and Development (ZonMw grants 917-86-319 to BBAdV, 916-86-016 to LELMV, 917-96-346 to AS and 907-00-365 to TK), the European Union under the 7th framework program (Gencodys HEALTH-F4-2010-241995 to BBAdV, HvB, ZA, AS and TK), the Dutch Brain Foundation (to DAK and BBAdV), the Hungarian Scientific Research Fund (K-82090 to ZA), and grants from the Jérôme Lejeune foundation and from the German Federal Ministry of Education and Research (BMBF funding to the German Mental Retardation Network, MRNET to AS).

\section{Competing interests None.}

Patient consent Obtained.

Ethics approval Local medical ethical committee.

Provenance and peer review Not commissioned; externally peer reviewed.

Web resources http://decipher.sanger.ac.uk; http://www.ecaruca.net

\section{REFERENCES}

van Bokhoven H. Genetic and epigenetic networks in intellectual disabilities. Ann Rev Genet 2011;45:81-104.

2 Kleefstra T, Kramer JM, Neveling K, Willemsen MH, Koemans TS, Vissers LE, Wissink-Lindhout W, Fenckova M, van den Akker WM, Kasri NN, Nillesen WM, Prescott T, Clark RD, Devriendt K, van Reeuwijk J, de Brouwer AP, Gilissen C, Zhou H, Brunner HG, Veltman JA, Schenck A, van Bokhoven H. Disruption of an
EHMT1-associated chromatin-modification module causes intellectual disability. Am J Hum Genet 2012;91:73-82.

$3 \mathrm{Ng} \mathrm{SB}$, Bigham AW, Buckingham KJ, Hannibal MC, McMillin MJ, Gildersleeve HI Beck AE, Tabor HK, Cooper GM, Mefford HC, Lee C, Turner EH, Smith JD, Rieder MJ, Yoshiura K, Matsumoto N, Ohta T, Niikawa N, Nickerson DA, Bamshad MJ, Shendure J. Exome sequencing identifies MLL2 mutations as a cause of Kabuki syndrome. Nat Genet 2010;42:790-3.

4 Hoischen A, van Bon BW, Gilissen C, Arts P, van Lier B, Steehouwer M, de Vries P, de Reuver R, Wieskamp N, Mortier G, Devriendt K, Amorim MZ, Revencu N, Kidd A, Barbosa M, Turner A, Smith J, Oley C, Henderson A, Hayes IM, Thompson EM, Brunner HG, de Vries BB, Veltman JA. De novo mutations of SETBP1 cause Schinzel-Giedion syndrome. Nat Genet 2010;42:483-5.

5 Hoischen A, van Bon BW, Rodriguez-Santiago B, Gilissen C, Vissers LE, de Vries P, Janssen I, van Lier B, Hastings R, Smithson SF, Newbury-Ecob R, Kjaergaard S, Goodship J, McGowan R, Bartholdi D, Rauch A, Peippo M, Cobben JM, Wieczorek D, Gillessen-Kaesbach G, Veltman JA, Brunner HG, de Vries BB. De novo nonsense mutations in ASXL1 cause Bohring-Opitz syndrome. Nat Genet 2011;43:729-31.

6 Sirmaci A, Spiliopoulos M, Brancati F, Powell E, Duman D, Abrams A, Bademci G, Agolini E, Guo S, Konuk B, Kavaz A, Blanton S, Digilio MC, Dallapiccola B, Young J, Zuchner S, Tekin M. Mutations in ANKRD11 cause KBG syndrome, characterized by intellectual disability, skeletal malformations, and macrodontia. Am J Hum Genet 2011;89:289-94.

7 Vissers LE, de Ligt J, Gilissen C, Janssen I, Steehouwer M, de Vries P, van Lier B, Arts $P$, Wieskamp N, del Rosario M, van Bon BW, Hoischen A, de Vries BB, Brunner HG, Veltman JA. A de novo paradigm for mental retardation. Nat Genet 2010;42:1109-12.

8 de Ligt J, Willemsen MH, van Bon BW, Kleefstra T, Yntema HG, Kroes T, Vulto-van Silfhout AT, Koolen DA, de Vries P, Gilissen C, del Rosario M, Hoischen A, Scheffer $H$, de Vries BB, Brunner HG, Veltman JA, Vissers LE. Diagnostic exome sequencing in persons with severe intellectual disability. $N$ Engl J Med 2012;367:1921-9.

9 Rauch A, Wieczorek D, Graf E, Wieland T, Endele S, Schwarzmayr T, Albrecht B, Bartholdi D, Beygo J, Di Donato N, Dufke A, Cremer K, Hempel M, Horn D, Hoyer J, Joset $P$, Röpke A, Moog U, Riess A, Thiel CT, Tzschach A, Wiesener A, Wohlleber E, Zweier C, Ekici AB, Zink AM, Rump A, Meisinger C, Grallert $H$, Sticht $H$, Schenck A, Engels H, Rappold G, Schröck E, Wieacker P, Riess O, Meitinger T, Reis A, Strom TM. Range of genetic mutations associated with severe non-syndromic sporadic intellectual disability: an exome sequencing study. Lancet 2012;380:1674-82.

10 Willemsen $M H$, Vissers LE, Willemsen MA, van Bon BW, Kroes T, de Ligt J, de Vries BB, Schoots J, Lugtenberg D, Hamel BC, van Bokhoven $H$, Brunner HG, Veltman JA, Kleefstra T. Mutations in DYNC1H1 cause severe intellectual disability with neuronal migration defects. J Med Genet 2012;49:179-83.

11 Feng Q, Zhang Y. The MeCP1 complex represses transcription through preferential binding, remodeling, and deacetylating methylated nucleosomes. Genes Dev 2011;15:827-32

12 Brackertz M, Gong Z, Leers J, Renkawitz R. p66alpha and p66beta of the Mi-2/ NuRD complex mediate MBD2 and histone interaction. Nucl Acids Res 2006;34:397-406.

13 Tsurusaki $Y$, Okamoto N, Ohashi H, Kosho T, Imai Y, Hibi-Ko Y, Kaname T, Naritomi K, Kawame H, Wakui K, Fukushima Y, Homma T, Kato M, Hiraki Y, Yamagata T, Yano S, Mizuno S, Sakazume S, Ishii T, Nagai T, Shiina M, Ogata K, Ohta T, Niikawa N, Miyatake S, Okada I, Mizuguchi T, Doi H, Saitsu H, Miyake N, Matsumoto N. Mutations affecting components of the SWI/SNF complex cause Coffin-Siris syndrome. Nat Genet 2012:44:376-8.

14 Kon C, Cadigan KM, da Silva SL, Nusse R. Developmental roles of the Mi-2/ NURD-associated protein p66 in Drosophila. Genetics 2005;169:2087-100.

15 Dietzl G, Chen D, Schnorrer F, Su KC, Barinova Y, Fellner M, Gasser B, Kinsey K, Oppel S, Scheiblauer S, Couto A, Marra V, Keleman K, Dickson BJ. A genome-wide transgenic RNAi library for conditional gene inactivation in Drosophila. Nature 2007;448:151-6.

16 Sharma P, Keane J, O'Kane CJ, Asztalos Z. Automated measurement of Drosophila jump reflex habituation and its use for mutant screening. J Neurosci Meth 2009;182:43-8

17 Asztalos Z, Arora N, Tully T. Olfactory jump reflex habituation in Drosophila and effects of classical conditioning mutations. J Neurogenet 2007;21:1-18.

18 Kramer JM, Kochinke K, Oortveld MA, Marks H, Kramer D, de Jong EK, Asztalos Z, Westwood JT, Stunnenberg HG, Sokolowski MB, Keleman K, Zhou H, van Bokhoven $\mathrm{H}$, Schenck $A$. Epigenetic regulation of learning and memory by Drosophila EHMT/G9a. PLOS Biol 2011;9:e1000569.

19 Koh YH, Gramates LS, Budnik V. Drosophila larval neuromuscular junction: molecular components and mechanisms underlying synaptic plasticity. Microsc Res Tech 2000;49:14-25.

20 Liu Z, Huang Y, Zhang Y, Chen D, Zhang YQ. Drosophila Acyl-CoA synthetase long-chain family member 4 regulates axonal transport of synaptic vesicles and is required for synaptic development and transmission. J Neurosci 2011;31:2052-63.

21 Schenck A, Bardoni B, Langmann C, Harden N, Mandel JL, Giangrande A. CYFIP/ Sra-1 controls neuronal connectivity in Drosophila and links the Rac1 GTPase pathway to the fragile X protein. Neuron 2003;38:887-98. 
22 Zweier C, de Jong EK, Zweier M, Orrico A, Ousager LB, Collins AL, Bijlsma EK, Oortveld MA, Ekici AB, Reis A, Schenck A, Rauch A. CNTNAP2 and NRXN1 are mutated in autosomal-recessive Pitt-Hopkins-like mental retardation and determine the level of a common synaptic protein in Drosophila. Am J Hum Genet 2009;85:655-66.

23 Koolen DA, Kramer JM, Neveling K, Nillesen WM, Moore-Barton HL, Elmslie FV, Toutain A, Amiel J, Malan V, Tsai AC, Cheung SW, Gilissen C, Verwiel ET, Martens S, Feuth T, Bongers EM, de Vries P, Scheffer H, Vissers LE, de Brouwer AP Brunner HG, Veltman JA, Schenck A, Yntema HG, de Vries BB. Mutations in the chromatin modifier gene KANSL1 cause the 17q21.31 microdeletion syndrome. Nat Genet 2012;44:639-41.

24 Koolen DA, Sharp AJ, Hurst JA, Firth HV, Knight SJ, Goldenberg A, Saugier-Veber P, Pfundt R, Vissers LE, Destrée A, Grisart B, Rooms L, Van der Aa N, Field M, Hackett A, Bell K, Nowaczyk MJ, Mancini GM, Poddighe PJ, Schwartz CE, Rossi E, De Gregori M, Antonacci-Fulton LL, McLellan MD II, Garrett JM, Wiechert MA, Miner TL, Crosby S, Ciccone R, Willatt L, Rauch A, Zenker M, Aradhya S, Manning MA, Strom TM, Wagenstaller J, Krepischi-Santos AC, Vianna-Morgante AM, Rosenberg C, Price SM, Stewart H, Shaw-Smith C, Brunner HG, Wilkie AO, Veltman JA, Zuffardi O, Eichler EE, de Vries BB. Clinical and molecular delineation of the 17q21.31 microdeletion syndrome. J Med Genet 2008;45:710-20.

25 Santen GW, Aten E, Sun Y, Almomani R, Gilissen C, Nielsen M, Kant SG, Snoeck IN, Peeters EA, Hilhorst-Hofstee Y, Wessels MW, den Hollander NS, Ruivenkamp CA, van Ommen GJ, Breuning MH, den Dunnen JT, van Haeringen A, Kriek M. Mutations in SWI/SNF chromatin remodeling complex gene ARID1B cause Coffin-Siris syndrome. Nat Genet 2012;44:379-80.

26 Van Houdt JK, Nowakowska BA, Sousa SB, van Schaik BD, Seuntjens E, Avonce N, Sifrim A, Abdul-Rahman OA, van den Boogaard MJ, Bottani A, Castori M, Cormier-Daire V, Deardorff MA, Filges I, Fryer A, Fryns JP, Gana S, Garavelli L, Gillessen-Kaesbach G, Hall BD, Horn D, Huylebroeck D, Klapecki J,
Krajewska-Walasek M, Kuechler A, Lines MA, Maas S, Macdermot KD, McKee S, Magee A, de Man SA, Moreau Y, Morice-Picard F, Obersztyn E, Pilch J, Rosser E, Shannon N, Stolte-Dijkstra I, Van Dijck P, Vilain C, Vogels A, Wakeling E, Wieczorek D, Wilson L, Zuffardi O, van Kampen AH, Devriendt K, Hennekam R, Vermeesch JR. Heterozygous missense mutations in SMARCA2 cause Nicolaides-Baraitser syndrome. Nat Genet 2012;44:445-9.

27 Wolff D, Endele S, Azzarello-Burri S, Hoyer J, Zweier M, Schanze I, Schmitt B, Rauch A, Reis A, Zweier C. In-frame deletion and missense mutations of the C-terminal helicase domain of SMARCA2 in three patients with Nicolaides-Baraitser Syndrome. Mol Syndromol 2012;6:237-44.

28 Kleefstra T, Brunner HG, Amiel J, Oudakker AR, Nillesen WM, Magee A, Geneviève D, Cormier-Daire V, van Esch H, Fryns JP, Hamel BC, Sistermans EA, de Vries $\mathrm{BB}$, van Bokhoven $\mathrm{H}$. Loss-of-function mutations in euchromatin histone methyl transferase 1 (EHMT1) cause the 9q34 subtelomeric deletion syndrome. Am J Hum Genet 2006;79:370-7.

29 Jones WD, Dafou D, McEntagart M, Woollard WJ, Elmslie FV, Holder-Espinasse M, Irving M, Saggar AK, Smithson S, Trembath RC, Deshpande C, Simpson MA. De novo mutations in MLL cause Wiedemann-Steiner syndrome. Am J Hum Genet 2012;91:358-64.

30 Clayton-Smith J, O'Sullivan J, Daly S, Bhaskar S, Day R, Anderson B, Voss AK, Thomas T, Biesecker LG, Smith P, Fryer A, Chandler KE, Kerr B, Tassabehji M, Lynch SA, Krajewska-Walasek M, McKee S, Smith J, Sweeney E, Mansour S, Mohammed S, Donnai D, Black G. Whole-exome-sequencing identifies mutations in histone acetyltransferase gene KAT6B in individuals with the Say-Barber-Biesecker variant of Ohdo syndrome. Am J Hum Genet 2011;89:675-81.

31 Marino S, Nusse R. Mutants in the mouse NuRD/Mi2 component P66alpha are embryonic lethal. Plos ONE 2007;2:e519.32.

32 Marhold J, Brehm A, Kramer K. The Drosophila methyl-DNA binding protein MBD2/ 3 interacts with the NuRD complex via p55 and MI-2. BMC Mol Biol 2004;5:20. 